\title{
Hepatic nuclear corepressor 1 regulates cholesterol absorption through a TR $\beta 1$-governed pathway
}

\author{
Inna Astapova, ${ }^{1}$ Preeti Ramadoss, ${ }^{1}$ Ricardo H. Costa-e-Sousa, ${ }^{1}$ Felix Ye, ${ }^{1}$ Kaila A. Holtz, ${ }^{1}$ \\ Yingxia Li,2 Michele W. Niepel,2 David E. Cohen,,2 and Anthony N. Hollenberg' ${ }^{1}$ \\ ${ }^{1}$ Division of Endocrinology, Diabetes and Metabolism, Beth Israel Deaconess Medical Center, Harvard Medical School, Boston, Massachusetts, USA. \\ 2Department of Medicine, Brigham and Women's Hospital, Harvard Medical School, Boston, Massachusetts, USA.
}

\begin{abstract}
Transcriptional coregulators are important components of nuclear receptor (NR) signaling machinery and provide additional mechanisms for modulation of NR activity. Expression of a mutated nuclear corepressor 1 (NCoR1) that lacks 2 NR interacting domains (NCoR $\triangle I D$ ) in the liver leads to elevated expression of genes regulated by thyroid hormone receptor (TR) and liver $X$ receptor (LXR), both of which control hepatic cholesterol metabolism. Here, we demonstrate that expression of NCoR $\triangle$ ID in mouse liver improves dietary cholesterol tolerance in an LXR $\alpha$-independent manner. NCoR $\triangle I D$-associated cholesterol tolerance was primarily due to diminished intestinal cholesterol absorption as the result of changes in the composition and hydrophobicity of the bile salt pool. Alterations of the bile salt pool were mediated by increased expression of genes encoding the bile acid metabolism enzymes CYP27A1 and CYP3A11 as well as canalicular bile salt pump ABCB11. We have determined that these genes are regulated by thyroid hormone and that TR $\beta 1$ is recruited to their regulatory regions. Together, these data indicate that interactions between NCoR1 and TR control a specific pathway involved in regulation of cholesterol metabolism and clearance.
\end{abstract}

\section{Introduction}

Cholesterol metabolism in the liver is critical for normal systemic regulation of serum cholesterol levels and eventual cardiac risk. Both the thyroid hormone receptor (TR) and liver $\mathrm{X}$ receptor (LXR) regulate multiple cholesterol clearance pathways and have been targeted as potential therapeutic avenues to improve cholesterol metabolism in humans (1-4). Thyroid hormone (TH) and its liver-specific analogs have been shown to lower serum cholesterol levels through increased expression of the LDL-R and/or HDL receptor SR-B1 in the liver $(2,5,6)$, stimulate cholesterol elimination via conversion to bile acids (3, 5, 7-9), enhance biliary cholesterol excretion through ATP-binding cassette transporter G5 and G8 (ABCG5/G8), and decrease cholesterol absorption (10-12). The effects of TH on the cholesterol metabolism in the liver are mediated by the TR $\beta 1$ isoform $(7,13)$.

LXR $\alpha$ and LXR $\beta$ are activated by oxysterols and act as intracellular cholesterol sensors (14). LXR $\alpha$ is the major isoform in the liver that plays a predominant role in maintaining hepatic cholesterol homeostasis. Global or liver-specific KO of Lxra leads to a dramatic hepatic cholesterol accumulation in mice fed diets with high cholesterol content (15-17). LXR $\alpha$ controls cholesterol clearance through regulation of intestinal cholesterol absorption and biliary cholesterol secretion as well as cholesterol conversion into bile acids $(15,17-19)$. Thus, TR $\beta 1$ and $\operatorname{LXR} \alpha$ regulate both distinct and overlapping pathways to control cholesterol metabolism in the liver. Based on this, we hypothesized that targeting common nuclear receptor (NR) coregulators could amplify beneficial effects of both pathways on cholesterol metabolism.

Previous work from our laboratory and others has demonstrated that the NR corepressor, nuclear corepressor 1 (NCoR1), is a criti-

Conflict of interest: The authors have declared that no conflict of interest exists. Citation for this article: J Clin Invest. 2014;124(5):1976-1986. doi:10.1172/JCI73419. cal regulator of hepatic metabolic function. We have shown that liver-specific expression of a Ncor1 allele coding for a protein that lacks the 2 NR interacting domains responsible for interactions with the TR and LXR (NCoR $\triangle \mathrm{ID}$ ) leads to the elevated expression of a number of enzymes involved in cholesterol and bile acid metabolism in these animals ( $L-\Delta I D$ mice) $(20)$. This observation together with the fact that LXR $\alpha$ and TR $\beta 1$ are critical for normal regulation of cholesterol metabolism prompted us to further examine the role of $\mathrm{NCoR} 1$ in this process.

Here, we demonstrate that hepatic expression of NCoR $\Delta$ ID leads to an improved cholesterol tolerance upon high-cholesterol feeding in both WT and cholesterol-intolerant LXR $\alpha$-deficient $\left(\mathrm{Lxra}^{-/}\right)$mice. This is largely due to changes in the bile acid pool composition that lead to decreased intestinal cholesterol absorption. Our results indicate that these effects are mediated by increased expression of bile acid synthesis and hydroxylation enzymes Cyp27a1 and Cyp3a11 as well as increased expression of canalicular bile salt pump $A b c b 11$ due to enhanced hepatic TR signaling. Thus, NCoR1 via its interactions with the TR controls a specific pathway to regulate cholesterol metabolism. Targeting of this pathway with therapeutics that specifically modify NCoR1 recruitment to the TR could provide substantial benefit to patients with hypercholesterolemia.

\section{Results}

Hepatocyte-specific expression of NCoR $\triangle I D$ reduces cholesterol deposition in the liver of mice fed with high-cholesterol diet independent of $L X R \alpha$. Our previous work demonstrated that expression of NCoR $\triangle \mathrm{ID}$ in the liver leads to the upregulation of expression of several enzymes in the cholesterol synthesis pathway $(20,21)$. To further investigate this, we examined cholesterol synthesis in this model. As shown in Figure 1A, the cholesterol synthesis rate as well as expression of Hmgcr, the rate-limiting enzyme in cholesterol synthesis path- 
A

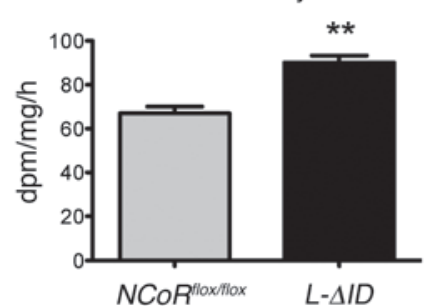

C Total hepatic cholesterol

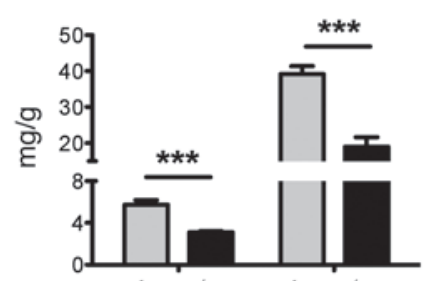

Lxra $^{+/+}$

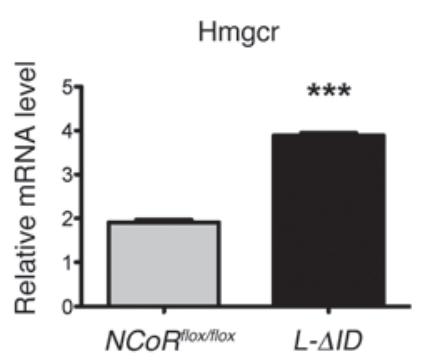

Esterified hepatic cholesterol

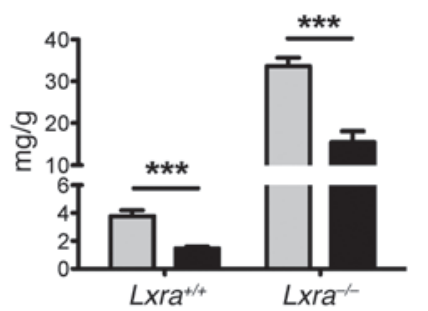

B
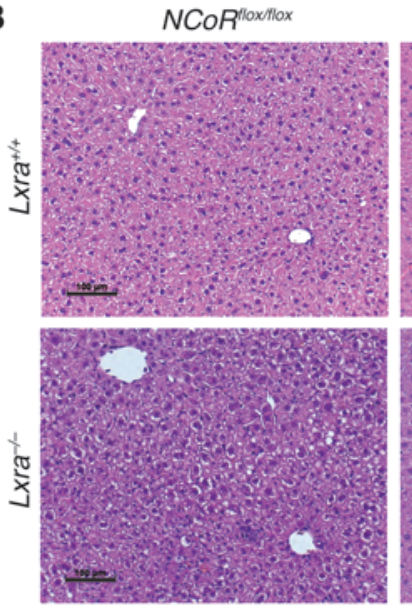
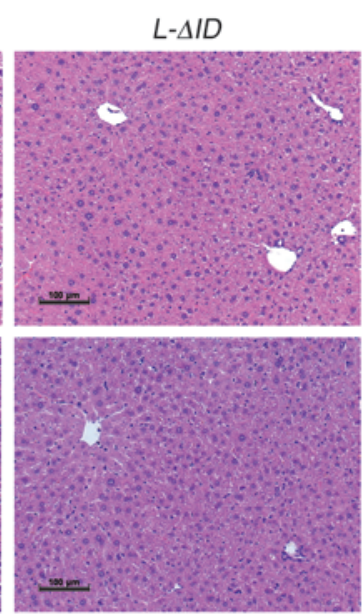

LXR effect $P<0.001$, no interaction

$N C O R^{\text {moxmilox }} \square-\Delta I D$

\section{Figure 1}

Hepatic cholesterol synthesis and deposition in $L-\Delta I D$ mice. (A) The rate of neutral sterol synthesis was assessed in primary hepatocytes isolated from $L-\Delta I D$ and control mice by incorporation of ${ }^{3} \mathrm{H}_{2} \mathrm{O}$. Shown are data from 1 representative experiment $(n=4$ wells per genotype). Expression of $\mathrm{Hmgcr}$ was measured by QPCR in the hepatocytes from the same experiment ( $n=3$ wells per genotype). (B) Representative H\&E-stained sections of livers from animals with indicated genotypes after 3 weeks on $2 \%$ cholesterol diet. Original magnification, $\times 20$. (C) Hepatic cholesterol content was measured in control and $L-\Delta I D$ mice on $L x \mathrm{xa}^{+/+}$and $L x \mathrm{ra}^{-/-}$background after 3 weeks on $2 \%$ cholesterol diet $(n=5-11$ animals per group). Statistical analysis was performed using unpaired Student's $t$ test $(\mathbf{A})$ or 2 -way ANOVA with Bonferroni post-tests $(\mathbf{C})$. ${ }^{* \star} P \leq 0.01 ;{ }^{* \star \star} P \leq 0.001$.

way, was significantly increased in primary hepatocytes isolated from $L-\Delta I D$ livers compared with controls, in agreement with the gene expression pattern observed in the whole liver (20). As a compensatory increase in cholesterol synthesis has been reported to be a result of increased cholesterol excretion in hyperthyroid rats (22), we hypothesized that the increase seen in $L-\Delta I D$ mice was also compensatory due to improved cholesterol clearance.

To gain further insight into the effects of NCoR $\Delta$ ID expression on hepatic cholesterol clearance and the role of TR and LXR $\alpha$ in NCoR $\Delta I D$ function in vivo, we crossed $L-\Delta I D$ and $\mathrm{Lxra}^{-/-}$mice and challenged them with high dietary cholesterol. The LXR $\alpha$ deficient mice were also chosen as an excellent model for this study due to their enhanced response to high-cholesterol feeding compared with WT mice. After 3 weeks of feeding with a $2 \%$ cholesterol-supplemented diet, we observed a 3-fold increase of total and esterified hepatic cholesterol in WT mice, but only a 1.5-fold increase in $L-\Delta I D$ mice (Figure $1, \mathrm{~B}$ and C). Remarkably, despite a further 6-fold increase in liver cholesterol content in cholesterolfed $L \times \mathrm{ra}^{-/-}$animals, which is in agreement with published data (15, 17), $L-\Delta I D \mathrm{Lxra}^{-/-}$mice still showed a dramatic reduction in hepatic cholesterol compared with $L x \mathrm{ra}^{-/-}$controls (Figure 1, B-C). This demonstrates that the absence of interactions between NCoR1 and NRs, such as the TR, has beneficial effects on hepatic cholesterol clearance that are independent of LXR $\alpha$.

NCoR $\triangle I D$ action in the liver is not proatherogenic. While we did not detect any differences in serum cholesterol concentrations between genotypes (Supplemental Table 1; supplemental material available online with this article; doi:10.1172/JCI73419DS1), plasma lipoprotein profiling showed, as expected, an increase in IDL/
LDL cholesterol in $\mathrm{Lxra}^{-/-}$animals (15). Additionally, it revealed a slight increase in VLDL cholesterol in $L-\Delta I D$ mice, which became more prominent on the $L \mathrm{xra}^{-/-}$background (Figure 2A). We next confirmed that hepatic expression of NCoR $\Delta$ ID was associated with an increased VLDL production rate by measuring serum triglyceride accumulation following the inhibition of lipoprotein lipase (Figure 2B). Thus, the export of cholesterol as part of VLDL particles is augmented in $L-\triangle I D$ mice, representing a potential route of cholesterol clearance from the liver (23). To examine whether secreted cholesterol could be deposited extra-hepatically, leading to the development of a proatherogenic phenotype, we measured whole-body cholesterol. Contrary to our expectations, we found that it was not increased in $L-\Delta I D$ mice and, moreover, was significantly diminished in $L-\Delta I D \mathrm{Lxra}^{-/-}$mice compared with $\mathrm{Lxra}^{-/-}$controls (Figure 2C). While we found that hepatic mRNA expression of $L d l r$ was upregulated in $L-\Delta I D$ mice on a $L x r a^{+/+}$background, this was not observed in $L-\Delta I D \mathrm{Lxra}^{-/-}$mice and could not account for the lower whole-body cholesterol in these animals. No differences were found in the hepatic expression of Idol (Mylip), which regulates LDL-R protein levels (24), HDL receptor SR-B1 (Scarb1), or circulating HDL levels, indicating that reduction in whole-body cholesterol content in $L-\Delta I D \mathrm{Lxra}^{-/-}$animals is unlikely to be due to increased reverse cholesterol transport (RCT). Thus, despite increased cholesterol secretion with VLDL, $L-\Delta I D$ mice do not store the cholesterol extrahepatically and appear to remove it through a mechanism separate from RCT.

Hepatic expression of NCoR $\triangle I D$ leads to an increase in fecal cholesterol elimination due to decreased intestinal cholesterol absorption. To further explore decreased hepatic and whole-body cholesterol 

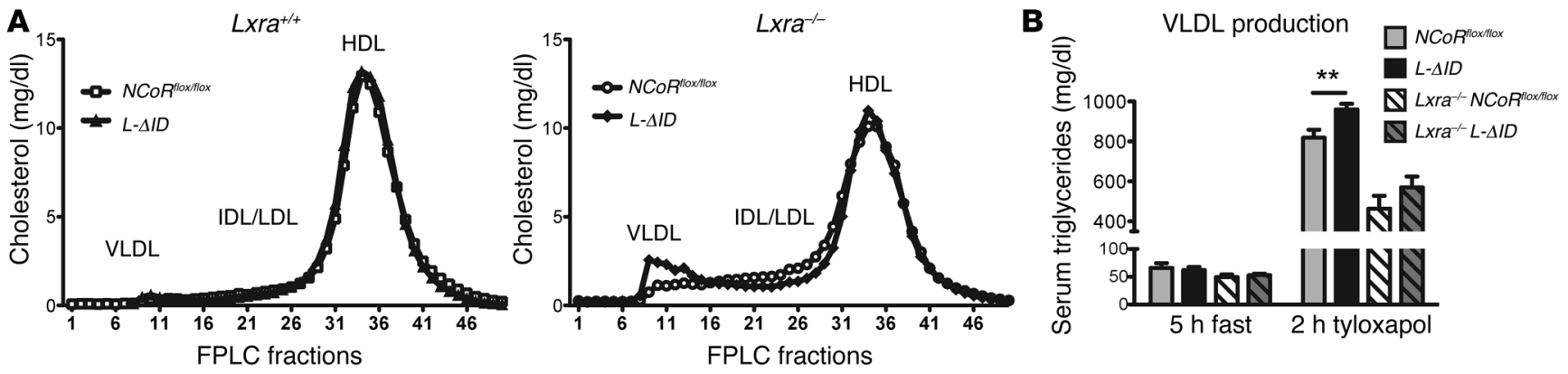

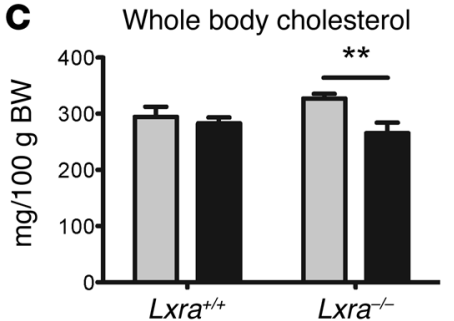

LXR effect $P<0.001$, no interaction

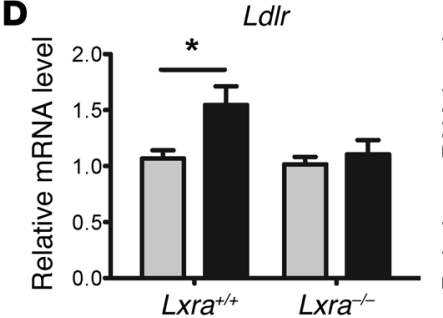

LXR effect $P<0.05$, no interaction

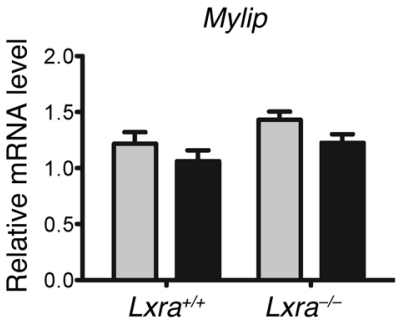

LXR effect $P<0.05$, no interaction

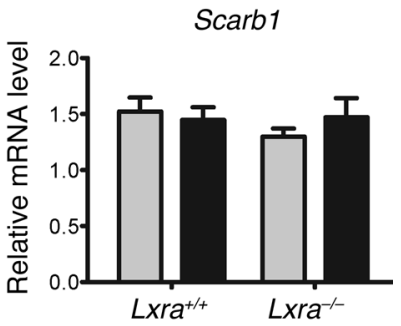

No LXR effect, no interaction

\section{Figure 2}

Effects of hepatic expression of NCoRAID on plasma lipoprotein and whole-body cholesterol content. (A) Cholesterol concentrations were measured in FPLC fractions of pooled plasma from animals with indicated genotypes fed a $2 \%$ cholesterol diet for 3 weeks $(n=6-9$ animals per group). (B) VLDL clearance was blocked in control and $L-\Delta I D$ mice on $L x \mathrm{xa}^{+/+}$and $L x \mathrm{xr}^{-/-}$backgrounds by intravenous injection of $600 \mathrm{mg} /$ $\mathrm{kg}$ tyloxapol. VLDL accumulation in blood was assessed by measuring plasma triglycerides at indicated time points. Animals were kept on highcholesterol diet for 3 weeks prior to the experiment $(n=5-8$ animals per group). (C) Whole carcasses (excluding liver and gut) of mice with indicated genotypes fed $2 \%$ cholesterol for 3 weeks were digested in ethanolic $\mathrm{KOH}$, and whole-body cholesterol content was measured $(n=5-8$ animals per group). (D) Expression of $L d l r$, Mylip (Idol), and Scarb1 was measured by QPCR in the livers of animals after 3 days of $2 \%$ cholesterol feeding ( $n=6-8$ animals per group). Statistical analysis was performed using 2 -way ANOVA with Bonferroni's post-tests. ${ }^{*} P \leq 0.05 ;{ }^{* *} P \leq 0.01$.

in $L-\Delta I D$ mice, we examined their fecal cholesterol output. The body weight, daily food intake, and fecal mass were not different between the genotypes after 3 weeks on a $2 \%$ cholesterol diet (Figure 3, A-C). In agreement with previously published observations, fecal cholesterol excretion was significantly decreased in $\mathrm{Lxra}^{-/-}$mice compared with $\mathrm{Lxra}^{+/+}$controls (17). Interestingly, introduction of hepatic NCoR $\Delta \mathrm{ID}$ almost normalized it, and a similar, but not significant, trend was seen in $L-\Delta I D$ mice on an $\mathrm{Lxra}^{+/+}$background (Figure 3D). The greater difference in fecal cholesterol output seen on the Lxra-/- background is most likely due to the greater cholesterol intolerance in these animals and a larger improvement and net reduction in the hepatic cholesterol deposition induced by NCoRAID expression in $\mathrm{Lxra}^{-/-}$animals (difference of $20 \mathrm{mg}$ of cholesterol per gram of liver tissue on $\mathrm{Lxra}^{-/-}$background vs. only $3 \mathrm{mg} / \mathrm{g}$ on $\mathrm{Lxra}^{+/+}$). Both LXR and TR have been shown to positively regulate fecal cholesterol output through upregulation of expression of $A b c g 5 / 8$ transporters, which leads to increased biliary cholesterol excretion $(11,12$, $17,19,25)$. Surprisingly, analysis of gall bladder bile revealed a decrease in biliary cholesterol concentration in $L-\Delta I D \mathrm{Lxra}^{+/+}$mice compared with controls and no differences in the $\mathrm{Lxra}^{-/-}$background (Figure $3 \mathrm{E}$ ). These changes were paralleled by decreased mRNA expression of $A b c g 5 / 8$ transporters (Figure $3 \mathrm{~F}$ ). While significant attenuation of expression of $A b c g 5 / 8$ transporters and biliary cholesterol excretion in $\mathrm{Lxra}^{-/-}$mice has been previously reported $(11,12,17,19,25)$, the changes observed in $\mathrm{Lxra}^{+/+}$ mice may be explained by the lack of LXR activation due to the decreased liver cholesterol content upon expression of NCoRAID.

As these findings indicate that enhanced fecal cholesterol elimination in $L-\Delta I D$ mice is not due to increased biliary excretion, we hypothesized that intestinal cholesterol absorption may be impaired in these animals. To directly test this, we employed the dual-isotope plasma method (26-28). Mice were given $1 \mu \mathrm{Ci}$ of ${ }^{14} \mathrm{C}$-labeled cholesterol intragastrically and $2.5 \mu \mathrm{Ci}$ of ${ }^{3} \mathrm{H}$-labeled cholesterol intravenously, and fractional cholesterol absorption was calculated based on the ratio of ${ }^{14} \mathrm{C}$ and ${ }^{3} \mathrm{H}$ present in plasma at 72 hours after tracer administration (Figure 3G). Indeed, intestinal cholesterol absorption was significantly decreased in $L-\Delta I D$ mice on an $\mathrm{Lxra}^{-/-}$background (27.3\% vs. $38.9 \%$ ) and trended in the same direction in $L-\Delta I D$ $\mathrm{Lxr}^{+/+}$mice compared with $\mathrm{Lxra}{ }^{+/+}$mice ( $\left.22.8 \% \mathrm{vs.} 30.7 \%\right)$, demonstrating that the differences in fecal cholesterol content are due to the changes in cholesterol absorption (Figure 3G).

Hepatic expression of NCoR $\triangle I D$ changes bile salt pool composition to decrease its bydrophobicity. To determine what factors are responsible for the decreased cholesterol absorption in $L-\Delta I D$ mice, we next examined bile acid metabolism. Bile salt pool size and composition are the major factors defining the rate of cholesterol absorption, with both decreased size and hydrophobicity leading to diminished cholesterol absorption (28-30). In addition, conversion of cholesterol into bile acids represents another pathway for cholesterol elimination from the liver and is positively regulated by LXR $\alpha$ and $\operatorname{TR} \beta 1(3,5,9,15,17,18)$. Interestingly, we found no difference in the 
A

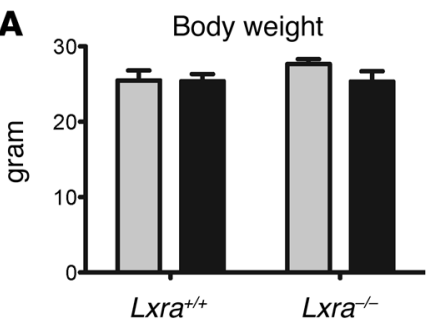

No LXR effect, no interaction

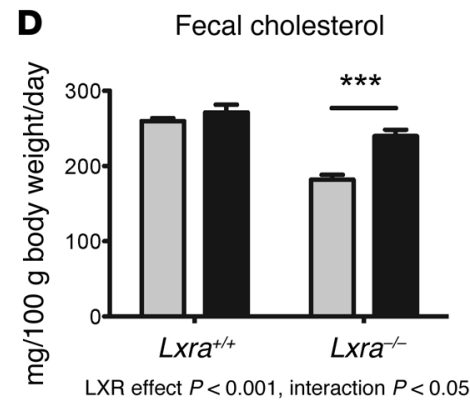

$\mathbf{F}$

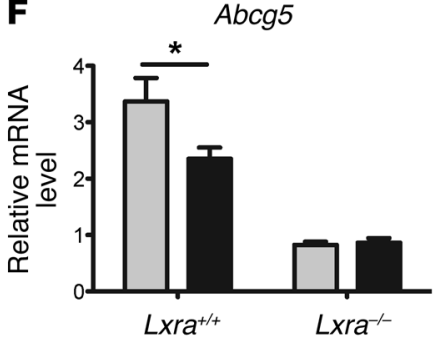

LXR effect $P<0.001$, no interaction

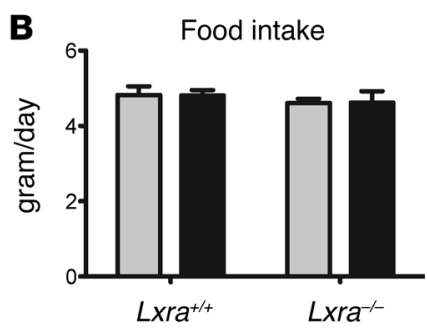

No LXR effect, no interaction

\section{$\mathbf{E}$}

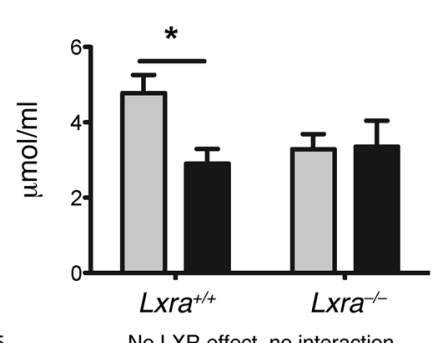

No LXR effect, no interaction

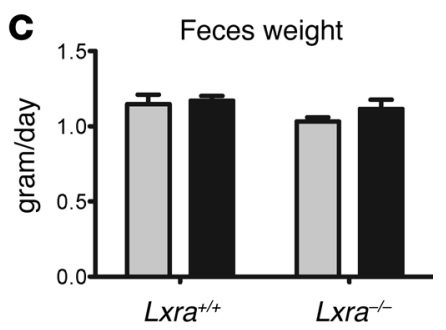

No LXR effect, no interaction

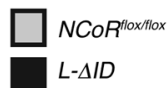

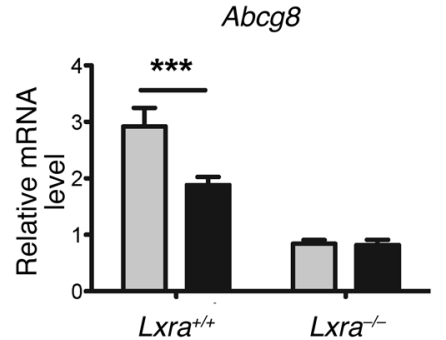

LXR effect $P<0.001$, interaction $P<0.05$

\section{G Cholesterol absorption}

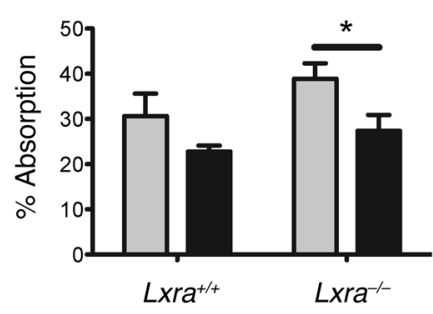

No LXR effect, no interaction

\section{Figure 3}

Effects of hepatic NCoRID on cholesterol excretion and intestinal cholesterol absorption. Body weight (A), daily food intake (B), daily fecal output (C), and fecal cholesterol concentrations (D) were measured in single caged mice over the 3 last days of the 3 weeks of high-cholesterol feeding ( $n=5-8$ animals per group). (E) Biliary cholesterol was measured in gall bladder bile of mice with indicated genotypes ( $n=3-8$ animals per group). (F) Hepatic expression of cholesterol transporters $A b c g 5 / 8$ in the animals with indicated genotypes $(n=5-11$ animals per group). (G) Fractional cholesterol absorption was measured in $L-\Delta / D$ and control animals on $L x \mathrm{ra}^{+/+}$and $L x \mathrm{xa}^{-/-}$background by dual-isotope plasma method. The data presented are calculated based on the 72-hour time point after tracer administration ( $n=5-9$ animals per group). All animals were fed a $2 \%$ cholesterol diet for 3 weeks prior to the experiments. Statistical analysis was performed using 2-way ANOVA with Bonferroni's post-tests. ${ }^{\star} P \leq 0.05$; ${ }^{\star \star \star} P \leq 0.001$.

levels of serum, hepatic, and biliary bile acids in $L-\Delta I D$ mice compared with controls on either a $\mathrm{Lxra}^{+/+}$or $\mathrm{Lxra}^{-/-}$background (Figure $4, \mathrm{~A}-\mathrm{C})$. Consistent with a key role of LXR $\alpha$ in the regulation of bile acid synthesis and elimination, hepatic bile acid concentrations and fecal bile salt excretion were significantly decreased in $\mathrm{Lxra}^{-/-}$ animals (Figure 4, B and D) (15). While total bile acid pool size was unchanged between the genotypes (Figure 4E), analysis of the pool composition revealed that, in agreement with previously published data, $\mathrm{Lxra}^{-/-}$animals had an elevated ratio of taurocholate to tauromuricholate (Figure 4F and Table 1), leading to an increased bile salt pool hydrophobicity index (Figure 4G), which results in an inappropriately high intestinal cholesterol absorption in these animals (15, 17). However, hepatic expression of NCoRAID led to a reduction in taurocholate and an increase in tauromuricholate levels independent of LXR $\alpha$ (Figure 4F and Table 1). This significant decrease in the bile salt pool hydrophobicity index (Figure 4G) led to decreased intestinal cholesterol absorption in $L-\Delta I D$ mice.
Upregulation of expression of alternative bile acid synthesis pathway enzymes mediates the decreased bile salt pool bydrophobicity seen in $L-\Delta I D$ mice. Effects of LXR and TR signaling on bile acid metabolism have been largely attributed to the upregulation of Cyp7a1 - a rate-limiting enzyme in the classic bile acid synthesis pathway $(3,5,7,9,15$, 18). Also, Cyp8b1, a $12 \alpha$-hydroxylase responsible for the synthesis of cholic acid (CA), has been shown to be downregulated by TH and LXR agonists $(17,31,32)$. The reduction in CYP8B1 activity leads to a decrease in synthesis of CA and a concomitant increase in production of more hydrophilic muricholic acid (MCA), a mechanism that is thought to underlie the LXR-mediated decrease in intestinal cholesterol absorption. Analysis of mRNA expression levels of enzymes implicated in bile acid synthesis, metabolism, and transport after 3 weeks of $2 \%$ cholesterol feeding (Supplemental Figure 1) showed that while some were affected by hepatic expression of NCoR $\Delta$ ID on $\mathrm{Lxra}^{+/+}$background, no significant differences could be seen in $\mathrm{Lxra}^{-/-}$animals. However, LXR $\alpha$ deficiency combined with 
A

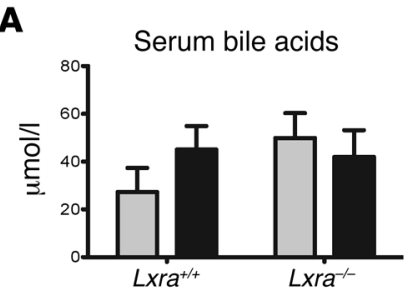

No LXR effect, no interaction

E

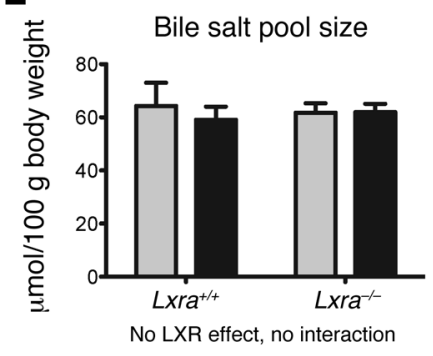

B

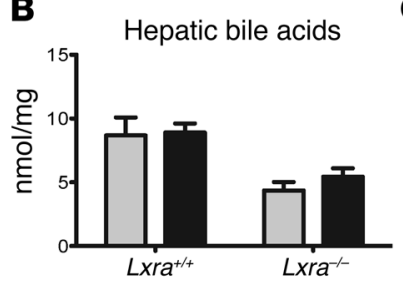

LXR effect $P<0.01$, no interaction

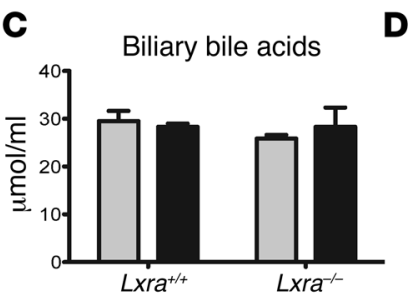

No LXR effect, no interaction

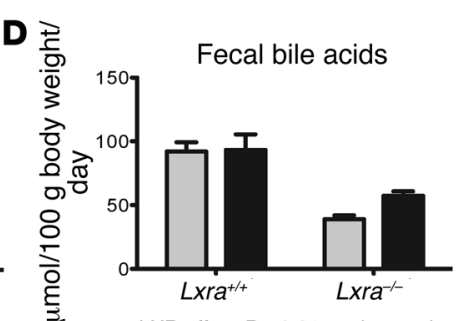

LXR effect $P<0.01$, no interaction
$\mathbf{F}$

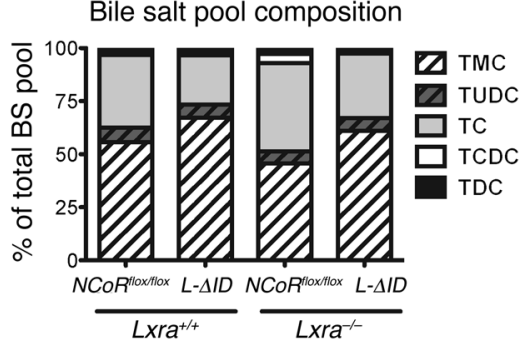

G

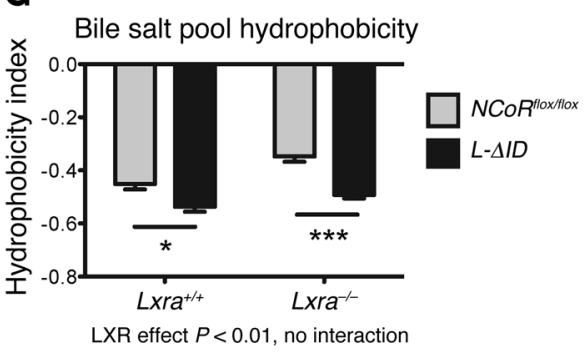

Figure 4

Bile acid metabolism in $L-\Delta I D$ mice. Total serum (A) $(n=6-10$ animals per group), hepatic (B) $(n=5-11$ animals per group), biliary $(\mathbf{C})$ ( $n=3-8$ animals per group), and fecal (D) ( $n=5-8$ animals per group) bile acid concentration in control and $L-\Delta / D$ animals on $L x \mathrm{r}^{+/+}$and $L x \mathrm{ra}^{-/-}$backgrounds. (E-G) Bile salts were extracted from liver, gall bladder, and small intestine that were collected from animals with the indicated genotypes. The size (E) and composition (F) were analyzed using HPLC and hydrophobicity index (G) was calculated by the method of Heuman (63). ( $n=4-8$ animals per group). TMC, tauromuricholate; TUDC, tauroursodeoxycholate; TC, taurocholate; TCDC, taurochenodeoxycholate; TDC, taurodeoxycholate. All animals were fed $2 \%$ cholesterol-supplemented chow for 3 weeks prior to the experiments. Statistical analysis was performed using 2-way ANOVA with Bonferroni's post-tests. ${ }^{*} P \leq 0.05$; ${ }^{* *} P \leq 0.001$.

3 weeks of cholesterol feeding resulted in a dramatic increase in cholesterol-induced liver damage, as indicated by elevated liver enzymes (Supplemental Table 1), and profound changes in the expression of most genes (Supplemental Figure 1). We therefore decided to assess expression of genes involved in bile acid synthesis after 3 days of $2 \%$ cholesterol feeding, when the effects of cholesterol diet in $\mathrm{Lxra}^{-/}$ mice are not yet complicated by liver damage and cholesterol accumulation. Surprisingly, we found that levels of Cyp7a1 or Cyp8b1 mRNA were not affected by the expression of NCoR $\Delta$ ID on either the $\mathrm{Lxa}^{+/+}$or $\mathrm{Lxra}^{-/-}$background (Figure 5A). Interestingly, mRNA expression of the first enzyme in the alternative bile acid synthesis pathway, Cyp27a1, was significantly upregulated in $L-\Delta I D$ mice independent of LXR $\alpha$ (Figure 5B). While there were no changes in the expression of another enzyme in the alternative pathway, Cyp7b1, we found that Cyp3a11, a major bile acid hydroxylation enzyme that has also been proposed to participate in alternative bile acid synthesis $(33,34)$, was dramatically upregulated in $L-\Delta I D$ animals. (Figure $5 \mathrm{~A})$. While expression of Cyp3a11 was significantly upregulated in $\mathrm{Lxra}^{-/}$animals, especially on the $2 \%$ cholesterol diet, indicating that LXR $\alpha$ and intracellular cholesterol levels play a role in its regulation, expression of NCoR $\triangle \mathrm{ID}$ led to additional upregulation, suggesting that a different NCoR1-dependent pathway is also involved (35). We also found that expression levels of liver bile salt export pump (BSEP, encoded for by $A b c b 11$ ) were elevated in $L-\Delta I D$ mice, while the mRNA level of Slc10a1 (coding for NTCP protein), the main transporter responsible for bile acid uptake into hepatocytes was unchanged (Figure 5A).

Importantly, mRNA expression of both Cyp3a11 and $A b c b 11$ was significantly elevated in $L-\Delta I D$ mice on either $L x \mathrm{ra}^{+/+}$or $\mathrm{Lxra}^{-/}$background fed a chow diet (Figure 5B) as well as in iso- lated primary hepatocytes (Figure 5C), suggesting a cell-autonomous effect of NCoR1. Levels of Cyp27a1 mRNA were also significantly upregulated in $L x \mathrm{ra}^{+/+} \mathrm{L}-\Delta I D$ chow-fed mice and primary hepatocytes (Figure 5, B-C). Thus, the upregulation of Cyp3a11 and Cyp27a1 in $L-\Delta I D$ mice is likely responsible for the changes in the bile salt pool composition and hydrophobicity index, while the increased expression of $A b c b 11$ may have a positive effect on bile acid flux and excretion.

Cyp27a1, Cyp3a11, and Abcb11 are TR $\beta$ targets. We have previously shown that NCoRAID augments LXR and TR signaling in the liver. Since the effects on cholesterol metabolism are LXR $\alpha$ independent and appear to be mediated by the regulation of the Cyp27a1, Cyp3a11, and Abcb11 genes, we next asked whether these genes are TH targets (Figure 6). Indeed, Cyp27a1 represents a classic positive $\mathrm{TH}$ target that is downregulated in hypothyroid mice and is significantly stimulated by T3 (Figure 6A and refs. 20, 36). Cyp3a11 is also regulated by T3 (37), but shows an atypical pattern with downregulation in the hypothyroid state and further suppression with T3 treatment (Figure 6A). Abcb11 expression was not affected by hypothyroidism, but T3 treatment led to its upregulation (Figure 6A). Since Cyp3a11 has been described as a CAR (Nr1i3) target gene (38) and $A b c b 11$ is a known FXR (Nr1b4) target (39), we examined expression of these NRs in L- $\Delta$ ID mice. Car (Nr1i3) mRNA was suppressed in $L-\Delta I D$ animals on all backgrounds, while expression of its prototypical target Cyp2b10 was not significantly changed (Supplemental Figure 2A). Neither Fxr $(\mathrm{Nr} 1 \mathrm{~h} 4)$ nor its target Shp (NrOb2) mRNA levels were affected in any of the genotypes examined, suggesting that these NRs are not responsible for the upregulation of expression of Cyp3a11 and Abcb11 (Supplemental Figure 2B). 
Table 1

Effects of hepatic NCoRsID expression on bile salt pool composition

\begin{tabular}{|c|c|c|c|c|}
\hline \multirow[b]{3}{*}{$\begin{array}{l}\text { Bile salt, } \\
\% \text { total pool }\end{array}$} & \multicolumn{4}{|c|}{ Genotype (n) } \\
\hline & \multicolumn{2}{|c|}{ Lxra $^{+/+}$} & \multicolumn{2}{|c|}{$\mathrm{Lxra}^{-/}$} \\
\hline & Ncorflox/flox (4) & $L-\Delta I D(7)$ & Ncorflox/flox (7) & $L-\Delta I D(5)$ \\
\hline TMC & $55.6 \pm 2.2$ & $67.2 \pm 2.2^{\mathrm{A}}$ & $45.6 \pm 1.4^{\mathrm{B}}$ & $61.0 \pm 1.5^{\mathrm{B}, \mathrm{C}}$ \\
\hline TUDC & $6.9 \pm 0.7$ & $6.2 \pm 0.3$ & $5.8 \pm 0.3$ & $6.1 \pm 0.4$ \\
\hline $\mathrm{TC}$ & $34.4 \pm 2.7$ & $23.2 \pm 2.3^{A}$ & $41.5 \pm 1.2^{\mathrm{D}}$ & $30.4 \pm 1.7^{A, D}$ \\
\hline TCDC & $1.8 \pm 0.3$ & $1.3 \pm 0.2$ & $4.3 \pm 1.5$ & $1.7 \pm 0.2$ \\
\hline TDC & $1.4 \pm 0.1$ & $2.1 \pm 1.0$ & $2.8 \pm 1.2$ & $0.9 \pm 0.1$ \\
\hline
\end{tabular}

Data are presented as mean \pm SEM. $n=5-7$ animals per group. ${ }^{A} P<0.01$, significant difference between $L-\Delta I D$ and $N c r^{\text {fibx } / \text { fibx }}$ on the same $L x$ ra background. ${ }^{B} P<0.001$

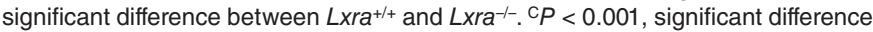
between $L-\Delta / D$ and Ncorflix ffibx on the same $L x$ ra background. $D P<0.01$, significant difference between $L x \mathrm{xa}^{+/+}$and $L \mathrm{xra}^{-/}$.

Deiodinases can have a significant impact on $\mathrm{TH}$ signaling within a specific tissue. Simultaneous disruption of Lxra and Lxrb combined with a Western diet results in a dramatic induction of hepatic mRNA expression and enzymatic activity of Dio2, which is normally not expressed in the liver (40). However, expression of Dio2 in all genotypes of mice in our study remained undetectable. We also found that, consistent with our previous findings, hepatic expression of NCoR $\Delta$ ID led to upregulation of Dio1 (20). $\mathrm{Lxra}^{-/-}$animals had higher expression of Dio1 compared with $\mathrm{Lxra}^{+/+}$controls, and while expression of Dio3 in adult mouse liver is very low, we found that it was further downregulated in $\mathrm{Lxra}^{-/}$ animals (Supplemental Figure 3A). Despite this, mRNA expression of hepatic TH targets, such as Bcl3, Gpd2, and Idh3 (Supplemental Figure 3B), was not affected in these animals, suggesting that changes in the expression levels of Diol and Dio3 in $\mathrm{Lxra}^{-/-}$mice are not physiologically important for TH signaling.

To further establish the role of TR signaling in the regulation of bile acid synthesis enzymes, we used a model recently developed and used for ChIP sequencing by our laboratory (41). To overcome the lack of reliable anti-TR antibodies, adenovirus was used to overexpress human TR $\beta 1$ tagged with a biotin ligase recognition peptide (Blrp) motif in the livers of mice that globally express the biotin ligase BirA. Crosslinked chromatin obtained from the livers of hypothyroid and hyperthyroid mice was used for precipitation using streptavidin beads followed by deep sequencing. We identified 3 TR $\beta 1$-binding peaks in the promoter and the first exon of the Cyp27a1 gene, 2 peaks in the proximal promoter and $5^{\prime}$ upstream region in the Cyp $3 a 11$ gene, and 2 peaks within promoter and intron 12 of the $A b c b 11$ gene (Figure 6C). We were able to confirm TR $\beta 1$ binding in these regions by performing standard ChIP-qPCR using primers located within the indicated regions (Figure 6B and Supplemental Table 2). Importantly, locations of these peaks align with the locations for NCoR1-binding peaks identified by Feng and coauthors (ref. 42 and Figure 6C). Thus, NCoR1 likely regulates expression of these bile acid synthesis and transport genes by recruitment via TR $\beta 1$, and its deletion would augment their expression.

\section{Discussion}

The role of TH signaling in the regulation of serum cholesterol levels has been well recognized since 1930 (43). As the liver plays a central role in maintaining cholesterol homeostasis, prog- ress has been made recently in the development of TR $\beta 1$ - and liver-specific thyromimetics that could potentially be used as cholesterol-lowering agents without undesirable effects that TH exerts on the heart and bone. However, the precise mechanisms of their action remain incompletely understood $(2-5,9)$. Interestingly, many of the effects of TR activation in the liver overlap with effects of LXR agonists, which suggests that there may be potential advantages in activation of both pathways $(15,17,19,25)$.

In addition to the use of specific ligands, modulation of interactions between NRs and their cofactors presents another opportunity to activate or repress NR actions. For example, liver-specific activation of the coactivator SRC-2 leads to an increase in FXRmediated stimulation of bile acid secretion that facilitates fat absorption and regulates energy intake (44). Muscle-specific and adipocyte-specific deletion of NCoR1 also leads to the activation of certain NR pathways in these tissues $(45,46)$. NCoR1 has been shown to bind both TR $\beta$ and $\operatorname{LXR} \alpha$ and repress transcription of their target genes in the presence of ligands $(20,47,48)$. Indeed, mice with hepatocyte-specific and whole-body expression of NCoR $\Delta$ ID show upregulation of expression of a number of TR and LXR target genes and demonstrate increased TH sensitivity in the liver and other tissues $(20,21)$.

We thus hypothesized that disruption of interactions between NCoR1 and NRs in the liver would improve cholesterol clearance through activation of TR and/or LXR-regulated pathways and potentially reveal novel pathways that could be therapeutically targeted. As shown herein, $L-\Delta I D$ mice exhibit improved tolerance for high dietary cholesterol characterized by a 2 -fold reduction in hepatic cholesterol concentrations compared with control animals. This effect persists in $\mathrm{Lxra}^{-/-}$animals, suggesting a major role for TR signaling, as activation of LXR $\beta$ cannot compensate for the absence of hepatic LXR $\alpha$ (17). Interestingly, the positive effects of the hepatic NCoRAID mutation were even stronger on the $\mathrm{Lxra}^{-/-}$ background, which is most likely related to the overall exacerbated dietary cholesterol intolerance of these animals.

Our data demonstrate that the blunted response to highcholesterol feeding observed in $L-\triangle I D$ mice is due to markedly diminished intestinal cholesterol absorption, which is largely dependent on the size and composition of bile acid pool (28-30). Indeed, bile salt pool hydrophobicity was significantly decreased in cholesterol-fed $L-\Delta I D$ mice due to the decrease in the CA to MCA ratio on both $L x r a^{+/+}$and $L x \mathrm{ra}^{-/-}$backgrounds. Surprisingly, hepatic expression of NCoR $\Delta$ ID did not affect expression of Cyp8b1 or Cyp7a1, 2 enzymes in the bile acid synthesis pathway known to be regulated by both $\operatorname{TR} \beta$ and $\operatorname{LXR} \alpha$ $(3,5,7,9,15,17,18,32)$. Instead, we found that expression of 2 other enzymes implicated in bile acid synthesis, Cyp27a1 and Cyp3a11, was markedly upregulated in $L-\Delta I D$ animals.

CYP27A1 catalyzes the first step of the alternative bile acid synthesis pathway and also carries out 27-hydroxylation of bile acid intermediates in the classic synthesis pathway. Mutations of this gene in humans lead to the development of cerebrotendinous xanthomatosis (CTX), characterized by deposition of cholesterol and cholestanol in tendons and the brain (49). Cyp27a1 disruption in mice results in abnormalities in lipid and BA metabolism and, notably, severely reduced BA synthesis with an increase in CA-to-MCA 
A
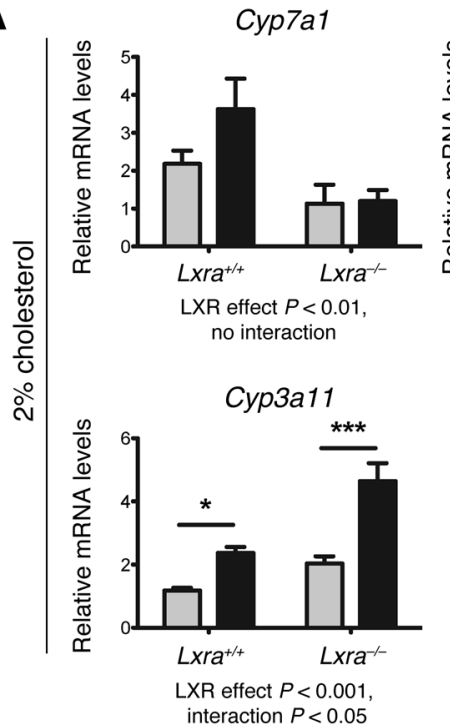

B

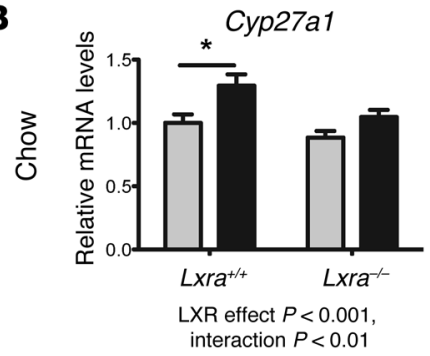

C

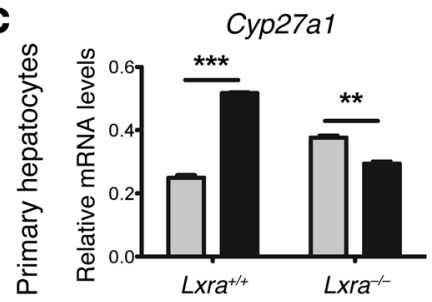

Cyp8b1

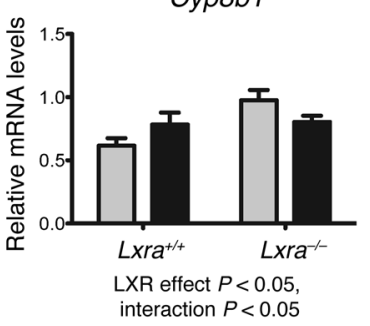

$A b c b 11$

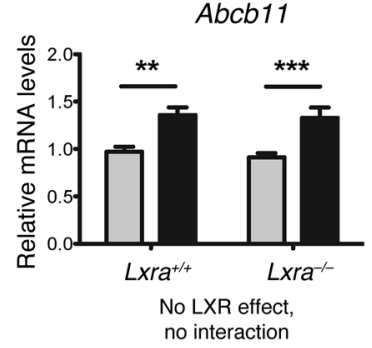

Сурза11

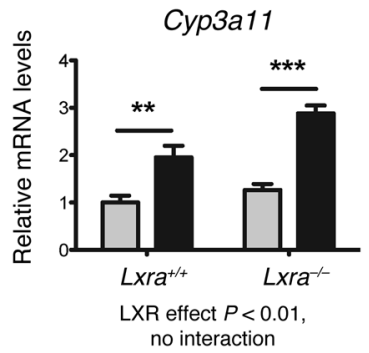

Сурза11

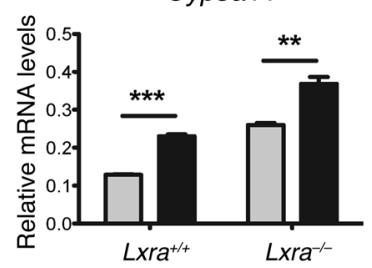

Cyp27a1

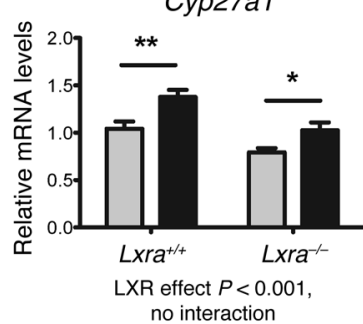

Slc10a1

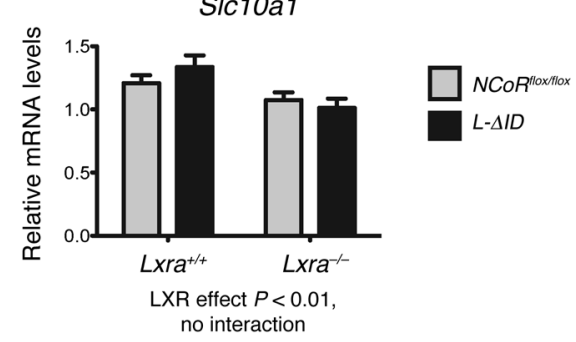

Abcb11

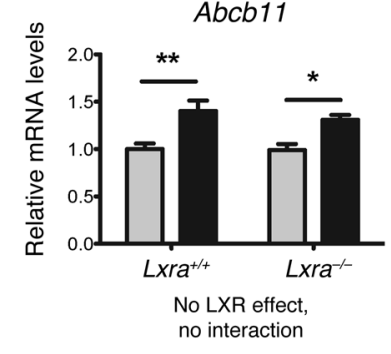

$A b c b 11$

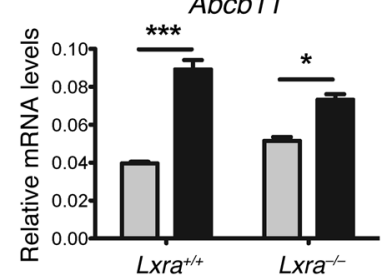

\section{Figure 5}

Hepatic expression of bile acid metabolism enzymes and transporters in $L-\Delta I D$ animals. mRNA expression levels of genes involved in bile acid synthesis, hydroxylation, and transport were quantified by QPCR $(\mathbf{A})$ in the livers of control and $L-\Delta I D$ animals on $L x \mathrm{xr}^{+/+}$and $L x \mathrm{xr}^{-/-}$backgrounds fed with $2 \%$ cholesterol diet for 3 days ( $n=7-9$ animals per group); (B) in the livers of chow-fed mice $(n=5-9$ animals per group); (C) in primary hepatocytes isolated from chow-fed animals with indicated genotypes. Shown are results of 1 experiment $(n=3$ wells per genotype). Statistical analysis was performed using 2-way ANOVA with Bonferroni's post-tests (A and B) or unpaired Student's $t$ test $(\mathbf{C})$ where $L x \mathrm{ra}^{+/+}$and $L x \mathrm{xa}^{-/-}$ hepatocytes were isolated on different days and no attempt was made to compare these 2 groups. ${ }^{\star} P \leq 0.05 ;{ }^{* \star} P \leq 0.01 ;{ }^{* \star} P \leq 0.001$.

ratio in the remaining pool (50-52). While hepatic overexpression of human Cyp27a1 in mice led to a generally mild phenotype, females fed a Western diet demonstrated an increase in fecal neutral sterol excretion and a decrease in CA-to-MCA ratio in biliary bile, consistent with our findings in L- $\Delta$ ID animals (53). While CYP3A11 is mainly known as a major drug-metabolizing P450 enzyme, there is evidence that it also plays a role in cholesterol and bile acid metabolism $(33,34)$. It has also been shown that highcholesterol feeding as well as $\operatorname{LXR} \alpha$ or $\operatorname{LXR} \alpha / \beta$ deficiency lead to dramatic upregulation of Cyp3a11 expression in mouse liver and overall Cyp3a11 mRNA levels correlate with the liver cholesterol content, suggesting that Cyp3a11 may mediate a compensatory response to cholesterol overload $(35,54)$. These data strongly suggest that upregulation of Cyp27a1 and Cyp3a11 expression in $L-\triangle I D$ animals is likely responsible for the observed changes in the bile acid pool composition and improved cholesterol tolerance.

In addition, expression of $A b c b 11$ (BSEP) was significantly upregulated in $L-\Delta I D$ mice. Mutations in this gene in humans lead to progressive familial intrahepatic cholestasis type 2, while mice deficient in $A b c b 11$ expression show a less profound cholestatic phenotype $(55,56)$. Mice with hepatic overexpression of $A b c b 11$ demonstrate increased biliary secretion of bile acids and cholesterol and 
A

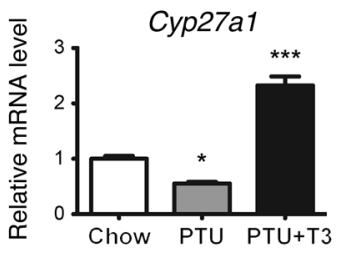

B

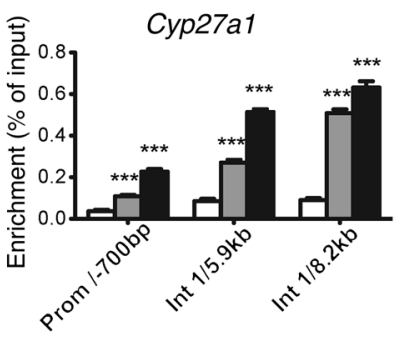

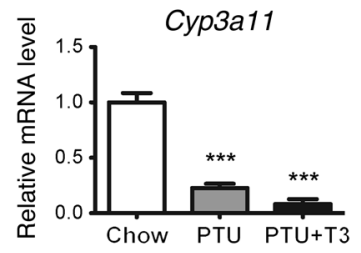

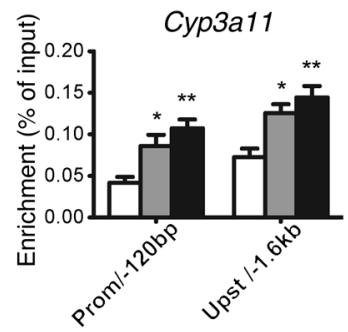

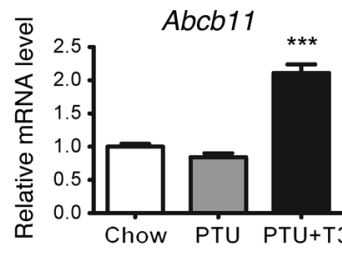

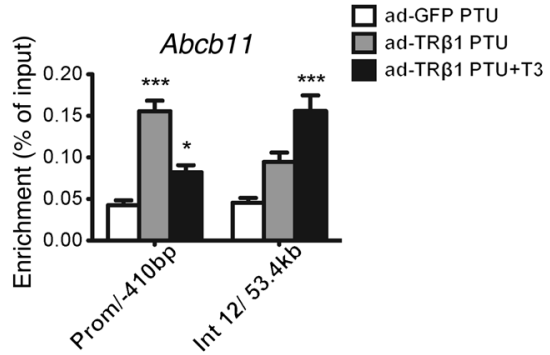

C
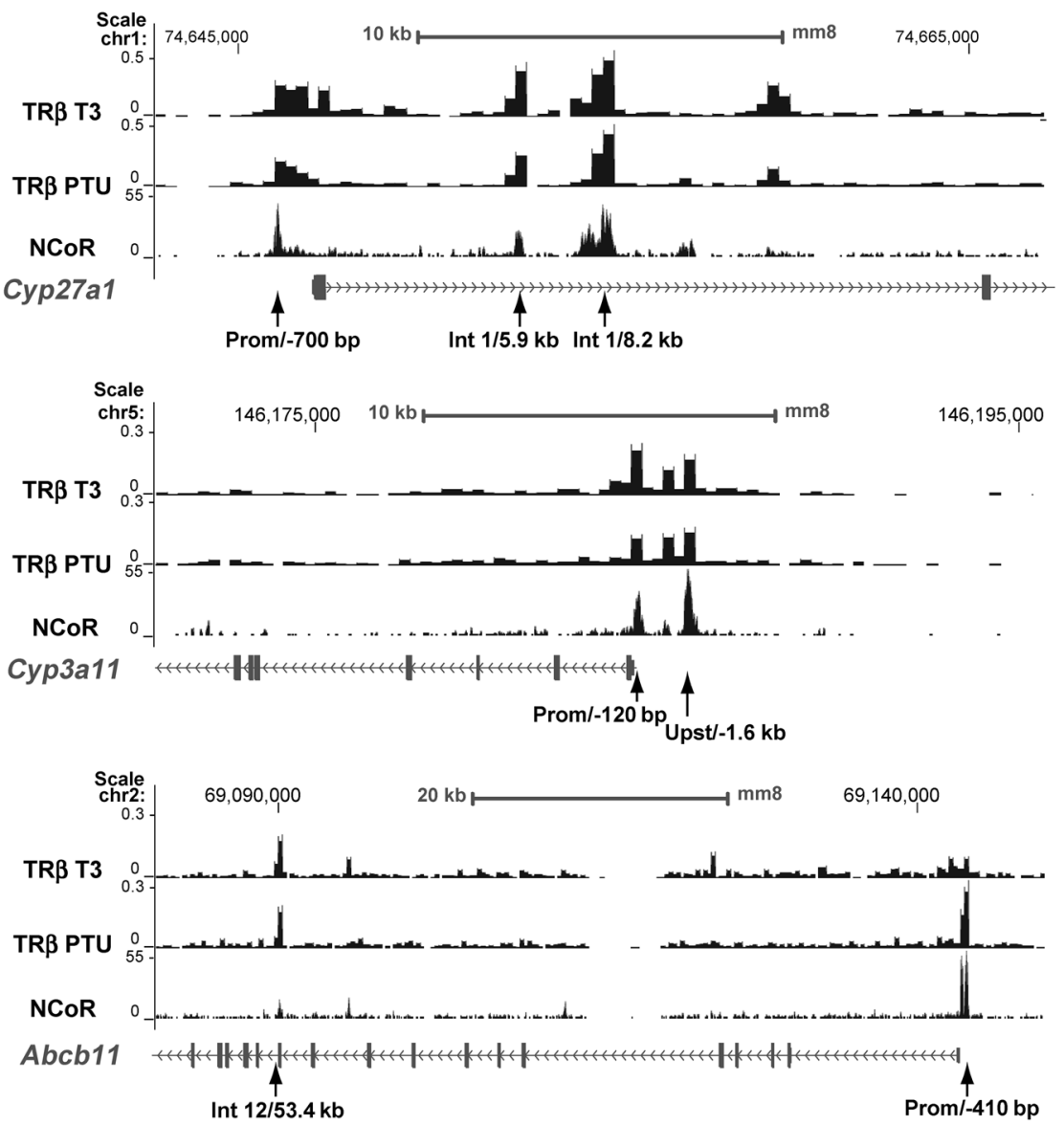

Figure 6

Cyp27a1, Cyp3a11, and Abcb11 are TR targets. (A) Expression of indicated genes was quantified by QPCR on RNA isolated from the livers of euthyroid (chow), hypothyroid (PTU), and hyperthyroid (PTU+T3) WT mice. ( $n=6-7$ animals per group). (B) ChIP followed by QPCR for indicated regions of Cyp27a1, Сyp3a11, and $A b c b 11$ genes was performed using streptavidin-agarose and chromatin isolated from livers of mice with hepatic adenovirus-mediated overexpression of GFP (control) or biotinylated TR $\beta 1(n=5$ affinity precipitation reactions per group). Statistical analysis was performed using 1-way ANOVA followed by Tukey's multiple comparisons test. ${ }^{*} P \leq 0.05$; ${ }^{* *} P \leq 0.01$; ${ }^{* * *} P \leq 0.001$. (C) Snapshots of UCSC genome browser showing alignment of histograms for TR ChIP peaks obtained in our laboratory (TR $\beta$ T3, TR $\beta$ PTU) and NCoR1 ChIP peaks (NCoR) obtained by Feng et al. (42) increased bile salt pool hydrophobicity due to elevated concentrations of taurodeoxycholate; they are also resistant to hepatic steatosis on high-fat and high-cholesterol diets supplemented with CA (57). While diminished hepatic cholesterol deposition in mice overexpressing $A b c b 11$ is similar to that in $L-\Delta I D$ animals, other features, such as increased BA pool hydrophobicity and increased biliary cholesterol excretion, are opposite of what is seen in $L-\Delta I D$ mice. It remains to be determined whether the elevated expression of $A b c b 11$ in $L-\Delta I D$ mice contributes to the decreased cholesterol accumulation or is a secondary response to diminished cholesterol absorption.
Since NCoR1 strongly regulates TH signaling in the liver and we did not see activation of CAR- or FXR-signaling pathways in $L-\triangle I D$ mice, we focused on the role of TH in regulating Cyp27a1, Cyp3a11, and $A b c b 11$ expression. We have found that mRNA expression levels of these genes are regulated by circulating $\mathrm{TH}$ and identified regions that directly bind the TR $\beta 1$ isoform within the promoter, upstream, and intronic regions of these genes by ChIP. Moreover, identified ChIP-sequencing peaks overlap with the regions that were demonstrated to bind NCoR1 (42), suggesting that the changes seen in the gene expression, par- 
ticularly in the absence of LXR $\alpha$, result from the lack of TRmediated recruitment of NCoR1 to these regulatory elements.

Taken together, our data demonstrate that disruption of NCoR1 function in the liver leads to improved dietary cholesterol tolerance through changes in bile acid metabolism that lead to decreased bile pool hydrophobicity and diminished intestinal cholesterol absorption. The data indicate that these changes are likely due to increased expression of 2 enzymes in the BA synthesis pathway - Cyp27a1 and Cyp3a11 - and potentially upregulation of the bile salt pump $A b c b 11$. In agreement with our previous findings that NCoR1 plays a key role in TR signaling in the liver, we have found that Cyp27a1, Cyp3a11, and $A b c b 11$ are TH targets and have identified TR-binding regions within the regulatory and coding regions of these genes, which coincide with the NCoR1-binding regions reported by others (42). Thus, disruption of NCoR1-TR interactions leads to increased expression of these target genes and improvement in cholesterol homeostasis. While this mechanism is most likely to be mediated by activation of the TR $\beta 1$-regulated pathway, a role for $\operatorname{TR} \alpha$ in it remains to be elucidated. However, this pathway is clearly distinct from those that are engaged not only by currently used TR $\beta 1$-specific agonists, but also by T3, which should activate both TR $\alpha$ and TR $\beta$. Thus, the differences between the effects of ligand-induced TR activation and lifting of NCoR1-mediated repression on cholesterol metabolism open a new avenue for therapeutic strategies with a potential to combine advantageous effects of activation of multiple NR, including TR and LXR.

\section{Methods}

Animal experiments. Alb-Cre Ncorflox/flox mice generated as described previously (20) were crossed to $\mathrm{Lxra}^{-/-}$mice (gift of David J. Mangelsdorf, Department of Pharmacology, University of Texas Southwestern Medical Center, Dallas, Texas, USA) to obtain $\mathrm{Lxra}^{+/-}$Alb-Cre Ncorflox/flox animals, which were used as breeders to generate $\mathrm{Lxra}^{+/+} \mathrm{N} \operatorname{cor} 1^{f l o x} /$ flox $($ control),

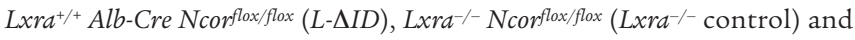
$\mathrm{Lxra}^{-/-}$Alb-Cre Ncorflox/flox $\left(\mathrm{Lxra}^{-/-} \mathrm{L}-\Delta I D\right)$ as littermates. Mice were maintained on mixed C57BL/6;129S background. Animals were housed at $22^{\circ} \mathrm{C}$ to $24^{\circ} \mathrm{C}$ on a 12 -hour light/12-hour dark cycle and given chow and water ad libitum. Details are provided in Supplemental Methods.

Histological analysis. Samples of livers were fixed in $4 \%$ formalin. Paraffin embedding, sectioning, and H\&E staining of the sections were performed using standard techniques at AML Labs Inc. (Baltimore, Maryland, USA).

Plasma lipids and liver enzymes. Total serum cholesterol, triglycerides, alanine aminotransferase (ALT), and aspartate aminotransferase (AST) were measured using standard assays purchased from Stanbio Laboratory. Serum bile acids were measured using 3- $\alpha$ HSD-based colorimetric Total Bile Acids Assay Kit (BIOQUANT).

Plasma lipoproteins in pooled samples from 6 to 9 animals per group were fractionated by fast protein liquid chromatography using a Superose 6 HR10/30 column (Amersham Biosciences), and cholesterol concentrations were determined in fractions as described previously $(58,59)$. Since the fractionation was performed on pooled samples, no statistical analysis of the results was performed.

In vivo VLDL production test. Mice were fasted for 5 hours and injected via tail vein with $600 \mathrm{mg} / \mathrm{kg}$ of Tyloxapol (Sigma-Aldrich) to block VLDL clearance. Blood samples were taken before and 2 hours after the injection. Plasma triglycerides were measured using enzymatic colorimetric assay from Stanbio Laboratory.

Whole-body cholesterol content. The extraction was performed as described by Bradley et al. (60). Briefly, mouse carcasses, excluding liver, gall bladder, stomach and small intestine, were each placed in a beaker containing 150 $\mathrm{ml}$ of ethanol and $10 \mathrm{~g} \mathrm{KOH}$ and allowed to autodigest for 4 days. After that, the contents were filtered and the volume was adjusted to $100 \mathrm{ml}$ with ethanol. The cholesterol concentrations were measured in the extracts using an enzymatic colorimetric kit from Stanbio.

Hepatic and biliary cholesterol and bile acid concentrations. The lipids were extracted by the method of Folch (61). Approximately $150 \mathrm{mg}$ of frozen livers or $5 \mu$ l of gall bladder bile was extracted in a chloroform:methanol $(2: 1)$ mixture. Following addition of $0.9 \% \mathrm{NaCl}$ and centrifugation, the upper methanol/water phase was used to measure bile acid concentrations with the Total Bile Acids Assay Kit (BIOQUANT). The organic phase was dried then dissolved in butanol/(Triton X-100:methanol [2:1]) (30:20); total and free cholesterol was measured using Cholesterol $\mathrm{E}$ and Free Cholesterol E Kits (Wako Chemicals). Esterified cholesterol concentrations were calculated as the difference between total and free cholesterol.

Fecal bile acid and cholesterol concentrations. The animals were single caged for 3 days, and the body weight and total food intake were recorded. At the end of this period, the feces were collected, dried, and weighed. Two 200-mg samples of the 3-day collection were used to extract and measure fecal bile acids and cholesterol. Total cholesterol was extracted by the Folch method as described above and measured using a colorimetric kit (Stanbio). Total bile acids were extracted in ethanol according to methods described by Locket and Gallaher (62) and measured with the Total Bile Acids Assay Kit (BIOQUANT).

Bile salt pool size and composition. The animals were fasted overnight and sacrificed by asphyxiation with $\mathrm{CO}_{2}$. The liver, gall bladder, and small intestine were excised en bloc, homogenized in $50 \mathrm{ml}$ of ethanol, and incubated at $60^{\circ} \mathrm{C}$ overnight. The extracts were spun down, and the liquid phase was dried and resuspended in methanol: $\mathrm{H}_{2} \mathrm{O}$ (75:25). Bile salt species were analyzed by HPLC using a Beckman Ultrasphere ODS column (4.6 $\mathrm{mm} \times 250$ $\mathrm{mm}, 5 \mu \mathrm{m}$; mobile phase methanol:0.01 $\mathrm{M} \mathrm{KH}_{2} \mathrm{PO}_{4}$ 75:25 vol:vol, pH 5.35) as previously described (59). Bile salt hydrophobic index was determined according to Heuman (63).

Intestinal cholesterol absorption. Cholesterol absorption was measured using the plasma dual isotope method as described (26-28). See Supplemental Methods for details.

Primary hepatocyte culture and cholesterol synthesis rate. Primary hepatocytes were isolated from 9- to 12-week-old male mice with indicated genotypes using standard collagenase digestion technique. Lipid synthesis rate was measured in hepatocyte cultures using the tritiated water method (64). Experiments were performed in at least 3 wells containing hepatocytes isolated from the same animals. See Supplemental Methods for details.

Real-time quantitative PCR. Real-time quantitative PCR (QPCR) was performed using standard procedures and TaqMan Gene Expression Assays purchased from Life Technologies. All data are presented as fold over expression in control $\mathrm{Lxra}^{+/+} \mathrm{Ncor} 1^{f l o x / f l o x} \mathrm{chow}$-fed group. See Supplemental Methods for details.

Generation of animals overexpressing biotinylated hTR $\beta 1$. The details of the study design, generation of the virus, and animal treatments are described in detail by Ramadoss et al. (41) and in Supplemental Methods.

ChIP assays. For each experiment, 4 livers per group of mice were used, and the chromatin was pooled before the affinity purification step. A total of 5 affinity precipitation reactions were carried out per each chromatin pool. The detailed protocol can be found in Ramadoss et al. (41) and Supplemental Methods. Primer sequences are provided in Supplemental Table 2.

Statistics. Statistical analysis was performed using Prism 5 program. The differences between genotypes were tested using 2-way ANOVA with Bonferroni's post-tests, 1-way ANOVA followed by Tukey's multiple comparison test, or unpaired 2-tailed Student's $t$ test where appropriate. All data are presented as mean \pm SEM. $P \leq 0.05$ was considered significant. 
Study approval. All animal experiments were approved by the Beth Israel Deaconess Medical Center Institutional Animal Care and Use Committee.

\section{Acknowledgments}

We thank David J. Mangelsdorf for $\mathrm{Lxra}^{-/-}$mice and Christopher K. Glass for the Blrp-TEV plasmid. This work was supported by NIH grants DK078090 and DK056123 (to A.N. Hollenberg), DK048873 and DK056626 (to D.E. Cohen), and the Harvard Digestive Diseases Center (P30 DK034854).
Received for publication October 9, 2013, and accepted in revised form February 13, 2014.

Address correspondence to: Anthony N. Hollenberg, 330 Brookline Ave. CLS-738, Boston, Massachusetts 02215, USA. Phone: 617.735.3268; Fax: 617.735.3323;E-mail: thollenb@bidmc.harvard.edu.

Ricardo H. Costa-e-Sousa's present address is: Institute of Biophysics Carlos Chagas Filho, Federal University of Rio de Janeiro, Rio de Janeiro, RJ, Brazil.
1. Calkin AC, Tontonoz P. Liver x receptor signaling pathways and atherosclerosis. Arterioscler Thromb Vasc Biol. 2010;30(8):1513-1518.

2. Erion MD, et al. Targeting thyroid hormone receptor-beta agonists to the liver reduces cholesterol and triglycerides and improves the therapeutic index. Proc Natl Acad Sci U S A. 2007;104(39):15490-15495.

3. Berkenstam A, et al. The thyroid hormone mimetic compound KB2115 lowers plasma LDL cholesterol and stimulates bile acid synthesis without cardiac effects in humans. Proc Natl Acad Sci U S A. 2008;105(2):663-667.

4. Ladenson PW, et al. Use of the thyroid hormone analogue eprotirome in statin-treated dyslipidemia. N Engl J Med. 2010;362(10):906-916.

5. Johansson L, et al. Selective thyroid receptor modulation by GC- 1 reduces serum lipids and stimulates steps of reverse cholesterol transport in euthyroid mice. Proc Natl Acad Sci U S A. 2005; 102(29):10297-10302.

6 . Tancevski I, et al. The liver-selective thyromimetic T-0681 influences reverse cholesterol transport and atherosclerosis development in mice. PLoS One. 2010;5(1):e8722.

7. Gullberg H, Rudling M, Forrest D, Angelin B, Vennstrom B. Thyroid hormone receptor betadeficient mice show complete loss of the normal cholesterol $7 \alpha$-hydroxylase (CYP7A) response to thyroid hormone but display enhanced resistance to dietary cholesterol. Mol Endocrinol. 2000;14(11):1739-1749.

8. Drover VA, Agellon LB. Regulation of the human cholesterol $7 \alpha$-hydroxylase gene (CYP7A1) by thyroid hormone in transgenic mice. Endocrinology. 2004;145(2):574-581

9. Lin JZ, et al. Thyroid hormone receptor agonists reduce serum cholesterol independent of the LDL receptor. Endocrinology. 2012;153(12):6136-6144.

10. Abrams JJ, Grundy SM. Cholesterol metabolism in hypothyroidism and hyperthyroidism in man. J Lipid Res. 1981;22(2):323-338.

11. Galman C, Bonde Y, Matasconi M, Angelin B, Rudling M. Dramatically increased intestinal absorption of cholesterol following hypophysectomy is normalized by thyroid hormone. Gastroenterology. 2008;134(4):1127-1136.

12. Bonde Y, Plosch T, Kuipers F, Angelin B, Rudling M. Stimulation of murine biliary cholesterol secretion by thyroid hormone is dependent on a functional ABCG5/G8 complex. Hepatology. 2012;56(5):1828-1837

13. Gullberg H, Rudling M, Salto C, Forrest D, Angelin B, Vennstrom B. Requirement for thyroid hormone receptor beta in $\mathrm{T} 3$ regulation of cholesterol metabolism in mice. Mol Endocrinol. 2002;16(8):1767-1777.

14. Janowski BA, Willy PJ, Devi TR, Falck JR, Mangelsdorf DJ. An oxysterol signalling pathway mediated by the nuclear receptor LXR $\alpha$. Nature. 1996;383(6602):728-731.

15. Peet DJ, et al. Cholesterol and bile acid metabolism are impaired in mice lacking the nuclear oxysterol receptor LXRa. Cell. 1998;93(5):693-704.

16. Alberti $S$, et al. Hepatic cholesterol metabolism and resistance to dietary cholesterol in LXR $\beta$-deficient mice. J Clin Invest. 2001;107(5):565-573.

17. Zhang Y, et al. Liver LXRalpha expression is crucial for whole body cholesterol homeostasis and reverse cholesterol transport in mice. J Clin Invest. 2012;122(5):1688-1699.

18. Chiang JY, Kimmel R, Stroup D. Regulation of cholesterol $7 \alpha$-hydroxylase gene (CYP7A1) transcription by the liver orphan receptor $(\operatorname{LXR} \alpha)$. Gene. 2001;262(1-2):257-265.

19. Repa JJ, Berge KE, Pomajzl C, Richardson JA, Hobbs H, Mangelsdorf DJ. Regulation of ATPbinding cassette sterol transporters ABCG5 and ABCG 8 by the liver $X$ receptors $\alpha$ and $\beta$. J Biol Chem. 2002;277(21):18793-18800.

20. Astapova I, Lee LJ, Morales C, Tauber S, Bilban M, Hollenberg AN. The nuclear corepressor, NCoR, regulates thyroid hormone action in vivo. Proc Natl Acad Sci U S A. 2008;105(49):19544-19549.

21. Astapova I, et al. The nuclear receptor corepressor (NCoR) controls thyroid hormone sensitivity and the set point of the hypothalamic-pituitary-thyroid axis. Mol Endocrinol. 2011;25(2):212-224.

22. Day R, et al. Time course of hepatic 3-hydroxy3-methylglutaryl coenzyme A reductase activity and messenger ribonucleic acid, biliary lipid secretion, and hepatic cholesterol content in methimazole-treated hypothyroid and hypophysectomized rats after triiodothyronine administration: possible linkage of cholesterol synthesis to biliary secretion. Endocrinology. 1989;125(1):459-468.

23. Vanpatten S, Karkanias GB, Rossetti L, Cohen DE. Intracerebroventricular leptin regulates hepatic cholesterol metabolism. Biochem J. 2004; 379(pt 2):229-233.

24. Zelcer N, Hong C, Boyadjian R, Tontonoz P. LXR regulates cholesterol uptake through Idol-dependent ubiquitination of the LDL receptor. Science. 2009;325(5936):100-104.

25. Yu L, York J, von Bergmann K, Lutjohann D, Cohen JC, Hobbs HH. Stimulation of cholesterol excretion by the liver $\mathrm{X}$ receptor agonist requires ATP-binding cassette transporters G5 and G8. J Biol Chem. 2003;278(18):15565-15570.

26. Turley SD, Herndon MW, Dietschy JM. Reevaluation and application of the dual-isotope plasma ratio method for the measurement of intestinal cholesterol absorption in the hamster. J Lipid Res. 1994;35(2):328-339.

27. Wang DQ, Carey MC. Measurement of intestinal cholesterol absorption by plasma and fecal dual-isotope ratio, mass balance, and lymph fistula methods in the mouse: an analysis of direct versus indirect methodologies. J Lipid Res. 2003;44(5):1042-1059.

28. Wang DQ, Tazuma S, Cohen DE, Carey MC. Feeding natural hydrophilic bile acids inhibits intestinal cholesterol absorption: studies in the gallstonesusceptible mouse. Am J Physiol Gastrointest Liver Physiol. 2003;285(3):G494-G502.

29. Cohen BI, Raicht RF, Mosbach EH. Sterol metabolism studies in the rat. Effects of primary bile acids (sodium taurochenodeoxycholate and sodium taurocholate) on sterol metabolism. J Lipid Res.
1977;18(2):223-231.

30. Hyogo H, Roy S, Paigen B, Cohen DE. Leptin promotes biliary cholesterol elimination during weight loss in ob/ob mice by regulating the enterohepatic circulation of bile salts. J Biol Chem. 2002;277(37):34117-34124.

31. Andersson U, Yang YZ, Bjorkhem I, Einarsson C, Eggertsen G, Gafvels M. Thyroid hormone suppresses hepatic sterol $12 \alpha$-hydroxylase (CYP8B1) activity and messenger ribonucleic acid in rat liver: failure to define known thyroid hormone response elements in the gene. Biochim Biophys Acta. 1999;1438(2):167-174.

32. Slatis K, et al. Abolished synthesis of cholic acid reduces atherosclerotic development in apolipoprotein E knockout mice. J Lipid Res. 2010;51(11):3289-3298.

33. Furster C, Wikvall K. Identification of CYP3A4 as the major enzyme responsible for 25-hydroxylation of $5 \beta$-cholestane- $3 \alpha, 7 \alpha, 12 \alpha$-triol in human liver microsomes. Biochim Biophys Acta. 1999;1437(1):46-52.

34. Honda A, et al. Cholesterol 25-hydroxylation activity of CYP3A. J Lipid Res. 2011;52(8):1509-1516.

35. Gnerre C, et al. LXR deficiency and cholesterol feeding affect the expression and phenobarbitalmediated induction of cytochromes $\mathrm{P} 450$ in mouse liver. J Lipid Res. 2005;46(8):1633-1642.

36. Yuan C, et al. Identical gene regulation patterns of T3 and selective thyroid hormone receptor modulator GC-1. Endocrinology. 2012;153(1):501-511.

37. Park YJ, Lee EK, Lee YK, Park do J, Jang HC, Moore DD. Opposing regulation of cytochrome P450 expression by CAR and PXR in hypothyroid mice. Toxicol Appl Pharmacol. 2012;263(2):131-137.

38. Hernandez JP, Mota LC, Huang W, Moore DD, Baldwin WS. Sexually dimorphic regulation and induction of $\mathrm{P} 450$ s by the constitutive androstane receptor (CAR). Toxicology. 2009;256(1-2):53-64.

39. Ananthanarayanan $M$, Balasubramanian $N$, Makishima M, Mangelsdorf DJ, Suchy FJ. Human bile salt export pump promoter is transactivated by the farnesoid $\mathrm{X}$ receptor/bile acid receptor.J Biol Chem. 2001;276(31):28857-28865.

40. Kalaany NY, et al. LXRs regulate the balance between fat storage and oxidation. Cell Metab. 2005;1(4):231-244.

41. Ramadoss P, et al. Novel mechanism of positive versus negative regulation by thyroid hormone receptor $\beta 1$ (TR $\beta 1$ ) identified by genome-wide profiling of binding sites in mouse liver. J Biol Chem. 2014;289(3):1313-1328.

42. Feng D, et al. A circadian rhythm orchestrated by histone deacetylase 3 controls hepatic lipid metabolism. Science. 2011;331(6022):1315-1319.

43. Mason RL, Hunt HM, Hurxthal L. Blood cholesterol values in hyperthyroidism and hypothyroidism - their significance. $N$ Engl J Med. 1930;203(26):1273-1277.

44. Chopra AR, et al. Cellular energy depletion resets whole-body energy by promoting coactivatormediated dietary fuel absorption. Cell Metab. 2011;13(1):35-43.

45. Yamamoto H, et al. NCoR1 is a conserved physiological modulator of muscle mass and oxidative 
function. Cell. 2011;147(4):827-839.

46. Li P, et al. Adipocyte NCoR knockout decreases PPAR $\gamma$ phosphorylation and enhances PPAR $\gamma$ activity and insulin sensitivity. Cell. 2011;147(4):815-826.

47. Wagner BL, et al. Promoter-specific roles for liver $\mathrm{X}$ receptor/corepressor complexes in the regulation of ABCA1 and SREBP1 gene expression. Mol Cell Biol. 2003;23(16):5780-5789.

48. Albers M, et al. A novel principle for partial agonism of liver $\mathrm{X}$ receptor ligands. Competitive recruitment of activators and repressors. J Biol Chem. 2006;281(8):4920-4930.

49. Cali JJ, Hsieh CL, Francke U, Russell DW. Mutations in the bile acid biosynthetic enzyme sterol 27-hydroxylase underlie cerebrotendinous xanthomatosis. J Biol Chem. 1991;266(12):7779-7783.

50. Rosen $\mathrm{H}$, et al. Markedly reduced bile acid synthesis but maintained levels of cholesterol and vitamin $\mathrm{D}$ metabolites in mice with disrupted sterol 27-hydroxylase gene. J Biol Chem. 1998; 273(24):14805-14812.

51. Repa JJ, et al. Disruption of the sterol 27-hydroxylase gene in mice results in hepatomegaly and hypertriglyceridemia. Reversal by cholic acid feeding. J Biol Chem. 2000;275(50):39685-39692.
52. Dubrac S, et al. Role of CYP27A in cholesterol and bile acid metabolism. J Lipid Res. 2005;46(1):76-85.

53. Meir K, et al. Human sterol 27-hydroxylase (CYP27) overexpressor transgenic mouse model. Evidence against 27-hydroxycholesterol as a critical regulator of cholesterol homeostasis. J Biol Chem. 2002;277(37):34036-34041.

54. Inoue S, Yoshinari K, Sugawara M, Yamazoe Y. Activated sterol regulatory element-binding protein-2 suppresses hepatocyte nuclear factor-4-mediated Cyp3a11 expression in mouse liver. Mol Pharmacol. 2011;79(1):148-156.

55. Strautnieks SS, et al. A gene encoding a liverspecific ABC transporter is mutated in progressive familial intrahepatic cholestasis. Nat Genet. 1998;20(3):233-238.

56. Wang R, et al. Targeted inactivation of sister of P-glycoprotein gene (spgp) in mice results in nonprogressive but persistent intrahepatic cholestasis. Proc Natl Acad Sci U S A. 2001;98(4):2011-2016.

57. Figge A, et al. Hepatic overexpression of murine Abcb11 increases hepatobiliary lipid secretion and reduces hepatic steatosis. J Biol Chem. 2004; 279(4):2790-2799.

58. Roy S, et al. A biphasic response of hepatobi- liary cholesterol metabolism to dietary fat at the onset of obesity in the mouse. Hepatology. 2005; 41(4):887-895.

59. VanPatten S, Ranginani N, Shefer S, Nguyen LB, Rossetti L, Cohen DE. Impaired biliary lipid secretion in obese Zucker rats: leptin promotes hepatic cholesterol clearance. Am J Physiol Gastrointest Liver Physiol. 2001;281(2):G393-G404.

60. Bradley MN, et al. Ligand activation of LXR $\beta$ reverses atherosclerosis and cellular cholesterol overload in mice lacking LXR $\alpha$ and apoE. J Clin Invest. 2007;117(8):2337-2346.

61. Folch J, Lees M, Sloane Stanley GH. A simple method for the isolation and purification of total lipides from animal tissues. J Biol Chem. 1957;226(1):497-509.

62. Locket PL, Gallaher DD. An improved procedure for bile acid extraction and purification and tissue distribution in the rat. Lipids. 1989;24(3):221-223.

63. Heuman DM. Quantitative estimation of the hydrophilic-hydrophobic balance of mixed bile salt solutions. J Lipid Res. 1989;30(5):719-730.

64. Bhatnagar S, Damron HA, Hillgartner FB. Fibroblast growth factor-19, a novel factor that inhibits hepatic fatty acid synthesis. J Biol Chem. 2009;284(15):10023-10033. 